\title{
Familial Bipolar Variant of Postpartum Psychosis: A Case Report
}

\author{
L Lavanya ${ }^{1}$, K Raman ${ }^{2}$, Shanthi Nambi ${ }^{3}$, GS Chandraleka ${ }^{4}$
}

\begin{abstract}
To highlight the familial influence in a patient presenting bipolar variant of postpartum psychosis (PP). Postpartum psychosis although less common than other postpartum psychiatric disorders is of immense significance in view of the course of the illness and impairment. Women with bipolar disorder or schizoaffective disorder have been reported to have $>50 \%$ risk for another episode of PP. Postpartum psychosis affected $74 \%$ of mothers with bipolar disorder and a first-degree relative who had PP, compared with only $30 \%$ of bipolar women without any family history of PP. There is a strong familial component associated with the illness. Mrs. S, 24-year-old housewife, married for 5 years, 10th std, from Puducherry, brought by the mother to our OPD with complaints of sleep disturbance, irritability, anger outburst over trivial issues, and suspiciousness for past 15 days. Stressor patient gave birth to a child 15 days back following which she developed sleep disturbances, decreased self-care, neglecting the child, suspiciousness that people are talking ill about her, and that her husband had left her permanently, with a past history revealing similar illness in the past during the first childbirth 3 years ago, which resolved within 10 days of treatment. Familial aggregation of the illness will be presented in detail and treatment options will be discussed.

Keywords: Bipolar disorder, Familial aggregation, Postpartum psychosis.

Indian Journal of Private Psychiatry (2019): 10.5005/jp-journals-10067-0036
\end{abstract}

\section{BACKGROUND}

Postpartum psychosis (PP) occurs in 1-2/1,000 of childbearing women.' Symptoms usually have an onset within the first 2-4 weeks after delivery. Diagnostic and statistical manual of mental disorders (DSM)-5 allows PP to be classified in the "short psychotic disorder" section of the schizophrenia spectrum and other psychotic disorders. But the preponderance of data suggests that PP is an overt presentation of bipolar disorder after delivery. ${ }^{1}$ 72-88\% of patients who develop PP immediately after childbirth have bipolar illness or schizoaffective disorder, whereas only $12 \%$ have schizophrenia. ${ }^{1}$ Close consanguinity increases the risk of mood disorders; morbidity risks for first-degree relatives are around $10-50 \%$ predisposing to puerperal psychosis.

The baseline risk for PP is 1:500; however, the risk rises to 1:7 for women with one past episode of PP. ${ }^{2}$ In fact, women with bipolar disorder or schizoaffective disorder have a $>50 \%$ risk for another episode of PP. ${ }^{3}$ Jones and Craddock ${ }^{4}$ found that PP affected $74 \%$ of mothers with bipolar disorder and a first-degree relative who had PP, compared with only $30 \%$ of bipolar women without any family history of PP. Women with a past history of unipolar psychotic depression can relapse shortly after delivery with an episode of PP.

Puerperal hormone shifts, obstetrical complications, sleep deprivation, and increased environmental stress are possible contributing factors to the onset of illness. ${ }^{5}$ As early as 2-3 days after childbirth, the patient develops paranoid, grandiose, or bizarre delusions, mood swings, confused thinking, and grossly disorganized behavior that represent a dramatic change from her previous functioning. PP and bipolar psychosis or mixed episodes share common symptoms of elation, dysphoria, mood lability, confusion, and heightened sensitivity to sleep deprivation.

\section{Case Description}

A 24-year-old female, Mrs. S, studied up to 10th standard, Muslim, Urdu speaking, middle socioeconomic status, and urban
${ }^{1-4}$ Department of Psychiatry, Saveetha Medical College and Hospital, Chennai, Tamil Nadu, India

Corresponding Author: K Raman, Department of Psychiatry, Saveetha Medical College and Hospital, Chennai, Tamil Nadu, India, Phone: +91 9789039260, e-mail: dr_ramkrish@yahoo.com

How to cite this article: Lavanya L, Raman K, Nambi S, et al. Familial Bipolar Variant of Postpartum Psychosis: A Case Report. Ind J Priv Psychiatry 2019;13(2):82-84.

Source of support: Nil

Conflict of interest: None

background was brought by mother with complaints of sleep disturbances, excessive talking, suspiciousness, decreased self-care, neglecting of the child, anger outburst, and irritability for the past 15 days. Onset was acute in nature, in the postpartum period, and course was progressive.

Patient was apparently normal 15 days back and she delivered a female baby by cesarean section in hospital. On the 3rd day of puerperium, she developed disturbed sleep, initially initial insomnia for few days, and changed to complete loss of sleep. She was constantly asking her mother about husband's presence. The hospital stay got prolonged for 2 weeks due to wound infection. During the hospital stay, she fed her baby every $2-3$ hours and maintained adequate self-care. After being discharged, during the 3rd week, she gradually neglected and stopped feeding her child. She started lamenting excessively about husband and her behaviour became odd and eccentric like repeatedly telling the names, scribbling, and collecting objects.

Seventeen days after delivery, she started expressing suspiciousness over husband's fidelity. Gradually, she started believing firmly that her husband had left her permanently for someone. She was not convinced by her husband's phone call or her mother's reassurance. Suspiciousness gradually involved her 
family members when she started believing that they were talking ill about her and preventing her from meeting her husband. She became restless and started pacing around the house. Behavioral changes continued to worsen and she started expressing anger and displayed aggression toward family members.

She was secluded in a room due to violent behavior and sleeplessness. She developed bladder and bowel incontinence initially for one day. She was brought to hospital by family members as she was not manageable at home.

There were no symptoms suggestive of schizophrenia or obsessive-compulsive disorder (OCD) or other anxiety disorders. There was no history of head injury or seizure disorder or suicidal attempt.

There is past history of similar illness soon after first childbirth 3 years ago. Symptoms during the previous episode were disturbed sleep pattern, decreased social interaction, decreased self-care, and food refusal. She was treated with psychotropic medications (details not available) and reached premorbid level in about 2 months.

\section{Family History}

In the 1st generation-patient's own sister had bipolar disorder and cousin sister had severe depression with psychosis. Her cousin brother had intellectual disability with psychosis unspecified. Patient's mother, two maternal aunts, and maternal uncle were diagnosed to have bipolar disorder. Patient's grandmother, grandmother's brother, and his daughter and son had bipolar disorder, and grandfather's elder brother and his three sons had psychiatric illness. There was history of thyroid dysfunction in three family members and recurrence of mental illness was associated with altered thyroid function in all of them. In family pedigree (Fig. 1), history of consanguinity was significantly noted. Each person in the family had predefined stressor before onset of their illness, and the duration of episode was shorter and symptoms remitted in few weeks with sodium valproate as the primary mood stabilizer in almost all patients. Evidence of completed suicide in two persons.

\section{Personal History}

Birth and developmental history were uneventful. She was average in studies, but discontinued in 10th standard due to fear of failure. She had regular cycles with no premenstrual mood changes. She is married for past 5 years and is currently staying with her in-laws and husband is working as assistant in attorney general office at Dubai. Interpersonal relationship with spouse is adequate. Premorbid personality did not reveal any abnormal

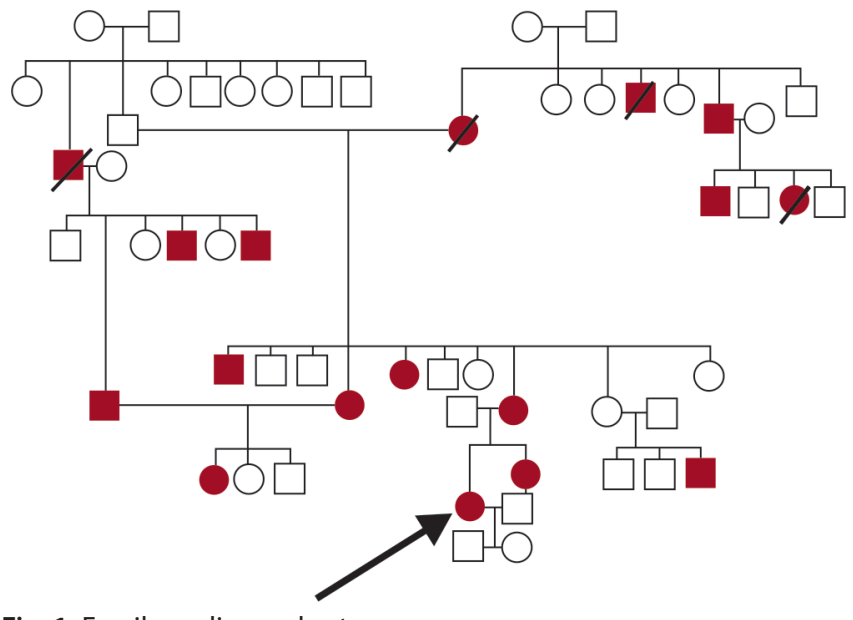

Fig. 1: Family pedigree chart personality traits. Her general and systemic examination did not reveal any abnormality. Mental state examination at the time of admission revealed increased psychomotor activity (restlessness and pacing around ward), difficult to establish rapport, speech was spontaneous, relevant and coherent, mood subjectively reported as worried, affect was irritable with thought content revealing delusion of reference and infidelity. There was no perceptual disturbance reported. Her higher mental functions showed impairment in abstract thinking and judgement with grade I insight.

To summarize, 24-year-old married female with significant family loading of affective disorder and family history of thyroid dysfunction with past history of depressive illness in the postpartum period with fair response to treatment presented with acute onset of behavioral change, paranoia, irritability, and insomnia in the postpartum period. Mental state examination revealed irritable affect, increased psychomotor activity, and paranoid delusions with poor insight.

A provisional diagnosis of mental or behavioral disorders associated with pregnancy, childbirth or the puerperium, with psychotic symptoms-6E21 was made as per ICD 10 and brief psychotic disorder with postpartum onset as per DSM 5. She was hospitalized in view of the unmanageability of symptoms. Her complete blood investigations were normal except for abnormal thyroid function test. Imaging was normal. She was started on Quetiapine $50 \mathrm{mg}$ titrated to $100 \mathrm{mg}$, lorazepam $4 \mathrm{mg}$ titrated to $8 \mathrm{mg}$, and sodium valproate $400 \mathrm{mg}$ titrated to $800 \mathrm{mg}$. Levothyroxine $25 \mu \mathrm{g}$ was started for hypothyroidism. She became symptom free within 2 months of treatment and is on regular follow-up. Psychoeducation of the family members regarding the illness, etiology, association with life events, treatment duration, prognosis, and child care was done in multiple sessions.

\section{Discussion}

The patient described here is a typical presentation of PP with the classical clinical picture of postpartum onset of sleep disturbance, paranoia, mood swings, and disorganized behavior. As it is reported in the literature, the patient presented with a clinical picture suggestive of mixed affective psychosis. She also had a past history of depressive illness which increased the risk for the current episode. Studies have reported that affective phenomenology seems to be a feature of the disorder and is more common in PP than other psychotic disorders. ${ }^{6}$ Apart from affective symptoms, at the same time, bizarre delirium-like symptoms may also be seen. Sometimes atypical cognitive symptoms such as disorientation and confusion can be observed in patients. Women have a relatively low incidence of symptoms such as thought insertion, withdrawal or broadcasting, passivity experiences, hallucinatory voices, giving running commentary, or social withdrawal., ${ }^{5,7}$

Rapid alterations in circulating steroid hormones following birth have consistently been suggested to play a role in PP etiology as have primiparity, maternal sleep problems, maternal stress in puerperium, and maternal age. ${ }^{4}$ Our patient had her first episode of postpartum mood disorder following her first childbirth. An affective episode with strong familial predisposition played a role in precipitation of illness. Although psychosocial factors play negligible role in the etiology, our patient had interpersonal conflicts with her in-law family and perceived absence of support from spouse possibly acting as stressful life events. It was interesting to note that the family members who reported of bipolar illness had precipitation of episodes following life events such as hearing death news, illness in a relative, and interpersonal conflicts at home. 
Postpartum psychiatric episodes may be a marker of a familial form of bipolar disorder. ${ }^{4}$ Familial aggregation was the highlight of this case study. We observed that there was strong genetic loading of affective illness across three generations. Since the whole family was being treated and was under follow-up with one psychiatrist for long duration, it was easy to delineate the pattern of illness and treatment response. Common findings observed include typical clinical presentation of life event triggering the onset of the illness, abrupt onset, agitated behavior and insomnia coupled with sad mood and irritable affect, and predominantly agitated depression like picture and faster remission to treatment with mood stabilizer (sodium valproate).

Thyroid dysfunction (hypothyroidism) was observed in our patient as comorbidity. We also observed similar pattern of thyroid abnormality in her family members who had affective illness. ${ }^{8,9}$ Studies have documented that autoimmune thyroid disease (thyroiditis) is much more prevalent in women with first-onset PP than in postpartum women from the general population. Further, clinical thyroid failure occurs significantly faster and in a greater percentage of patients with PP. Postpartum immune activation is postulated to produce the clinical manifestations of both thyroid dysfunction and psychiatric illness.

A recent study suggests that lithium prophylaxis is effective in women who previously had only PP than in women with bipolar disorder who have had mood episodes outside the postpartum period as well. Lithium, divalproex, olanzapine, and estrogen have been examined for their efficacy in preventing the recurrence of psychosis. Our patient had a significant response to sodium valproate, which was the first choice in view of the family response to treatment with the same drug. Valproate is effective as a mood stabilizer and is approved as safe for breastfeeding. Maintenance dose with valproate was effective in preventing recurrence in her first-degree relatives with bipolar affective disorder. ${ }^{10-12}$

Good prognostic factors included patients with a shorter duration of the acute episode of PP compared with patients with longer episodes and first onset affective psychosis outside postpartum period. ${ }^{13}$ Our patient had a better prognosis in terms of early treatment response and remission, typical clinical picture with no features of delirium, compliance with treatment, and family support.

\section{Conclusion}

The case was presented for its uniqueness in terms of strong familial risk and treatment response. We did not evaluate the patient for autoimmune thyroid antibodies which would have established clue to the role of thyroid dysfunction in the illness onset. The clinical presentation was a prototype of PP with predominant affective disturbance, past affective episode with postpartum onset, and early remission with treatment. Hence, it is imperative to screen patients with genetic, biological, and social risk factors for postpartum exacerbation of mental illness.

\section{Clinical Significance}

The above case description highlights the familial aggregation of mood disorder, episode association with life events, link between thyroid dysfunction and recurrence of mood disorder, and importance of familial treatment response.

\section{References}

1. Kendell RE, Chalmers JC, Platz C. Epidemiology of puerperal psychoses. Br J Psychiatry 1987;150:662-673. DOI: 10.1192/ bjp.150.5.662.

2. Sit D, Rothschild JA, Wisner KL. A Review of Postpartum Psychosis. J Womens Health 2006;15(4):352-368. DOI: 10.1089/jwh.2006. 15.352.

3. Davies W. Understanding the pathophysiology of postpartum psychosis: Challenges and new approaches. World J Psychiatry 2017;7(2):77-88. DOI: 10.5498/wjp.v7.i2.77.

4. Jones I, Craddock N. Familiality of the Puerperal Trigger in Bipolar Disorder: Results of a Family Study. Am J Psychiatry 2001;158(6): 913-917. DOI: 10.1176/appi.ajp.158.6.913.

5. Jones I, Chandra PS, Dazzan P, et al. Bipolar disorder, affective psychosis, and schizophrenia in pregnancy and the post-partum period. Lancet 2014;384(9956):1789-1799. DOI: 10.1016/S01406736(14)61278-2.

6. Bokhari R, Bhatara VS, Bandettini F, et al. Postpartum psychosis and postpartum thyroiditis. Psychoneuroendocrinology 1998;23(6): 643-650. DOI: 10.1016/S0306-4530(98)00034-1.

7. MacKinnon AL, Naguib M, Barr HJ, et al. Delusional ideation during the perinatal period in a community sample. Schizophr Res 2017;179: 17-22. DOI: 10.1016/j.schres.2016.09.027.

8. Jones I, Smith S. Puerperal psychosis: identifying and caring for women at risk. Adv Psychiatr Treat 2009;15(6):411-418. DOI: 10.1192/ apt.bp.107.004333.

9. Chakrabarti S. Thyroid Functions and Bipolar Affective Disorder. J Thyroid Res 2011;2011:306367. DOI: 10.4061/2011/306367.

10. Doucet S, Jones I, Letourneau N, et al. Interventions for the prevention and treatment of postpartum psychosis: a systematic review. Arch Womens Ment Health 2011;14(2):89-98. DOI: 10.1007/s00737-0100199-6.

11. Bergink V, Bouvy PF, Vervoort JS, et al. Prevention of postpartum psychosis and mania in women at high risk. Am J Psychiatry 2012;169(6):609-615. DOI: 10.1176/appi.ajp.2012.11071047.

12. Sharma V. Pharmacotherapy of postpartum psychosis. Expert Opin Pharmacother 2003;4(10):1651-1658. DOI: 10.1517/14656566.4.10.1651.

13. Chaudron LH, Pies RW. The relationship between postpartum psychosis and bipolar disorder: a review. J Clin Psychiatry 2003;64(11):1284-1292. DOI: 10.4088/JCP.v64n1102. 